\title{
The pseudo-symmetric $N$-benzyl hydroxyethylamine core in a new series of heteroarylcarboxyamide HIV-1 Pr inhibitors: synthesis, molecular modeling and biological evaluation.
}

\author{
Rosarita D’Orsi, ${ }^{a}$ Maria Funicello, ${ }^{a}$ Paolo Lupattelli, ${ }^{a}$ Federico Berti, ${ }^{*}, \mathrm{~b}$ and Lucia Chiummiento*,a \\ a Dipartimento di Scienze, Università della Basilicata, Via Ateneo Lucano 10, 85100 Potenza, Italy. \\ ${ }^{\mathrm{b}}$ Dipartimento di Scienze Chimiche e Farmaceutiche, Università di Trieste, via Giorgieri, 1, 34127 \\ Trieste, Italy. fberti@units.it; lucia.chiummiento@unibas.it
}

Keywords: HIV-protease inhibitors; pseudo-symmetric core, heteroaryl carboxyamides; synthesis, biological screening; modeling.

\begin{abstract}
:
New series of compounds containing both heterocycle moieties and pseudo-symmetric hydroxyethylamine core were obtained using a simple synthetic path that can provide a library of compounds in few steps and high yields. Furthermore, diversity-oriented synthesis was studied to change different functionalities according to needs. The in vitro inhibition activity against recombinant HIV-1 protease was evaluated. A beneficial effect of this class of compounds can be obtained either for the presence of a bis-benzyl group into the core and for the heterocyclic moiety in P1, specifically the indole ring. Docking analysis was also reported.
\end{abstract}

\section{Introduction}

The AIDS epidemic is still one of the most challenging problems [1], although great efforts are made to the discovery of new drugs for its treatment. Among many strategies to combat the disease, anti-retroviral therapy (ART) containing at least one of HIV-1 protease inhibitors (PIs) is considered as the most effective treatment [2-5]. When a protease inhibitor binds the active site, it prevents cleavage of nascent viral proteins, thereby halting viral replication [6]. The synthesis of compounds able to block the action of the HIV protease, the enzyme which plays a key role in maintaining infectivity, is currently a huge aim.

Nowadays, nine approved PIs are available on the market, but due to the rapid genomic evolution of the HIV, an inevitable consequence in the treatment of the infection has been the rise of drug resistance and therefore the dramatic reduction of the marketed inhibitors efficiency [7, 8].

Thus, the emergence of highly mutated viral strains cross-resistant to antivirals, the occurrence of various side effects, the high cost of ART, prompted scientists to seek novel PIs, desirably with alternative frameworks. 
Notably, introduction of heterocyclic moieties in a bioactive molecule can have important effects on physicochemical and pharmacological properties [9]. This strategy has been widely adopted in medicinal chemistry for the design of new drugs, because of their chemical stability and structural rigidity less entropic energy was lost upon binding. In our experience of synthesis of highly functionalized small molecules with aryl and heteroaryl structures [10-12] we evidenced the crucial effect of the presence of heterocyclic moiety in PIs structure either in the core of the inhibitors [1315] or in $\mathrm{P} 2$ position [16-18].

Inspired by the last commercially available inhibitors and its analogue, Darunavir and TMC-126, which showed inhibition of the dimerization and inhibition of proteolytic activity (figure 1), our objective was focused on designing inhibitors containing heterocyclic moieties that specifically target and maximize interactions with the backbone. Both extensive hydrogen bonding and hydrophobic interactions with enzyme subsites can limit the protease ability to acquire drug resistance as the geometry of the catalytic site must be conserved to maintain functionality [19-21]. The preparation and the activity, in vitro and in mammalian cells, of new HIV protease inhibitors, compounds 1 and 2, were recently described. They were designed having heterocycle as P2 ligand linked by a carboxyamidic or carbammic moiety to the core, with or without the benzyl group, and a 3,4-dimethoxyphenylsulfonyl- $N$-isobutylamide [22] or a 4-methoxyphenylsulfonyl- $N$ isobutylamide [23] as P2' ligand (figure 1). Compounds with benzyl in the core showed in vitro activity against native protease, with $\mathrm{IC}_{50}$ values in the range of $<0.6-13 \mathrm{nM}$.
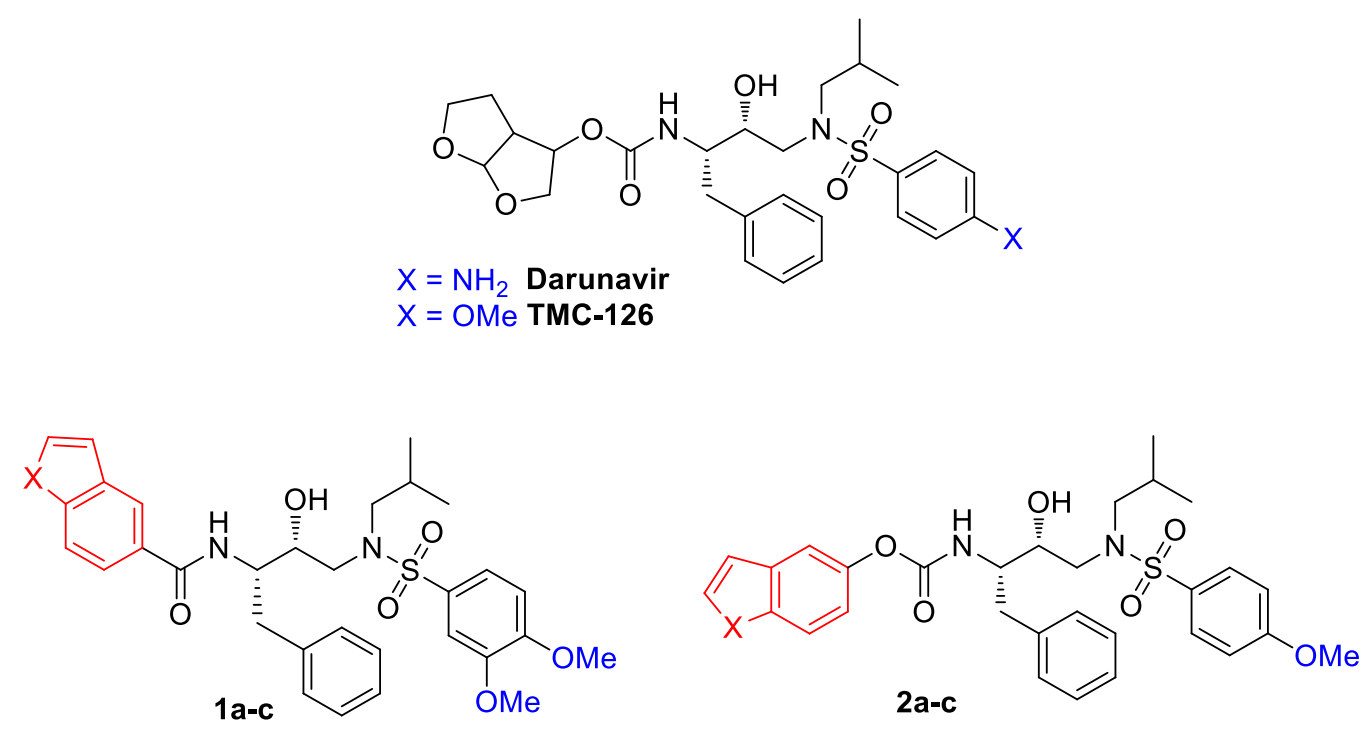

$\left(\mathrm{IC}_{50}=1-13 \mathrm{nM}\right)$

a: $X=S ; \mathbf{b}: X=O ; \mathbf{c}: X=N H$

$\left(\mathrm{IC}_{50}<0.6-11 \mathrm{nM}\right)$

Figure 1. Commercial and not HIV-Protease inhibitors. 
As HIV protease has been shown to exist as a C2-symmetric homodimer in its active form, several dipeptide isosteres such as diaminoalcohol, diaminodiol and hydroxyethylhydrazine have been also employed in the development of pseudo-symmetric inhibitors [24] (Figure 2).<smiles>CCN[C@@H](Cc1ccccc1)C(O)CC(Cc1ccccc1)NC</smiles>

diaminoalcohol<smiles>CN[C@@H](Cc1ccccc1)[C@H](O)[C@H](O)[C@@H](Cc1ccccc1)NC</smiles>

diaminodiol

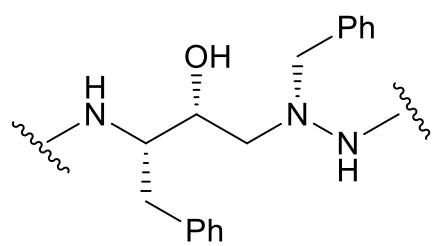

hydroxyethylhydrazine<smiles>[R]c1ccc(S(=O)(=O)N(Cc2ccccc2)[C@H](O)[C@H](Cc2ccccc2)NC(=O)c2ccc3[X]ccc3c2)cc1</smiles>

Figure 2. Examples of pseudosymmetrical core of HIV-protease inhibitors.

For this purpose a library of compounds containing different heterocycles and sulfonamide portions were prepared. In particular, in order to obtain a pseudo-symmetric hydroxyethylamine core the isobutyl portion presents in the structure of compounds $\mathbf{1}$ and $\mathbf{2}$ was replaced with a benzyl group. The effects on inhibitory activity of the heteroatom $(\mathrm{S}, \mathrm{O}, \mathrm{N})$ in the heteroarylcarboxamidic portion and the electronic properties of the substituents on the sulfonamidic moiety were also evaluated. The general structure of these compounds is reported as A in figure 2.

\section{Materials and Methods}

\subsection{Chemistry}

Preparative chromatography was carried out on Merck silica gel $(0.063-0.200 \mathrm{~mm}$ particle size) by progressive elution with opportune solvent mixtures. ${ }^{1} \mathrm{H}$ and ${ }^{13} \mathrm{C}$ NMR spectra were normally carried out in $\mathrm{CDCl}_{3}$ solutions on a VARIAN INOVA $500 \mathrm{MHz}$ or Bruker $400 \mathrm{MHz}$ and referenced to $\mathrm{CDCl}_{3}$. Mass spectra were obtained with a Hewlett-Packard 5971 mass-selective detector on a Hewlett-Packard 5890 gas chromatograph [(OV-1 capillary column between 70 and $250{ }^{\circ} \mathrm{C}\left(20{ }^{\circ} \mathrm{C}\right.$ $\left.\min ^{-1}\right)$ ]. The optical purity was evaluated by using a polarimeter JASCO Mod Dip-370. $\mathrm{CH}_{2} \mathrm{Cl}_{2}$ was dried by distillation over anhydrous $\mathrm{CaCl}_{2}$ in inert atmosphere. Dry THF and DMF were commercially available. 
tert-butyl (2S,3R)-4-(benzylamino)-3-hydroxy-1-phenylbutan-2-ylcarbamate (4). Compound 4 was prepared from a solution of of (2S,3S)-1,2-epoxy-3-(Boc-amino)-4-phenylbutane (1.5 mmol) and benzylamine $(1.5 \mathrm{mmol})$ in $i$-PrOH $(10 \mathrm{~mL})$ that was stirred under reflux for $16 \mathrm{~h}$. The reaction mixture was rotary evaporated, and the crude product was purified by recrystallization in methanol/water (7:3). Compound 4 was obtained as a white solid, yield $88 \% .{ }^{1} \mathrm{H}$ and ${ }^{13} \mathrm{C}$ NMR spectra were consistent to literature data [25].

General procedure for the preparation of tert-butyl $((2 S, 3 R)-N-[4-(N-b e n z y l-4-R-$ phenylsulfonamido)-3-hydroxy-1-phenylbutan-2-yl)carbamates (5). To a stirred solution of aminoalcohol $4(0.78 \mathrm{mmol})$ in anhydrous $\mathrm{CH}_{2} \mathrm{Cl}_{2}(20 \mathrm{~mL}), \mathrm{Et}_{3} \mathrm{~N}(2.02 \mathrm{mmol})$ and arylsulfonyl chloride $(0.93 \mathrm{mmol})$ were added at room temperature and under Ar atmosphere. After $24 \mathrm{~h}$ the reaction was quenched with $5 \%$ aqueous $\mathrm{H}_{2} \mathrm{SO}_{4}$ solution. The organic layer was washed adding saturated aqueous $\mathrm{NaHCO}_{3}$ solution and brine. The organic phases collected were dried over $\mathrm{Na}_{2} \mathrm{SO}_{4}$, filtered and concentrated in vacuo. The crude was purified by column chromatography on silica gel.

(1S,2R)-\{1-Benzyl-2-hydroxy-3-[N-benzyl-(4-nitrobenzenesulfonyl)-amino]-propyl\}-carbamic acid tert-butyl ester (5a). Compound $5 \mathbf{a}$ was isolated as white solid $\left(\mathrm{CH}_{2} \mathrm{Cl}_{2} / \mathrm{EtOAc} 98 / 2\right)$, yield 94 \%. $[\alpha]_{D}^{20}=+6.3^{\circ}\left(\mathrm{c}: 1.0, \mathrm{CHCl}_{3}\right) .{ }^{1} \mathrm{H}$ NMR (400 MHz, DMSO-d 6 ): $\delta 8.37(\mathrm{~d}, J=9.0 \mathrm{~Hz}, 2 \mathrm{H})$, $8.10(\mathrm{~d}, J=9.0,2 \mathrm{H}), 7.33(\mathrm{~m}, 5 \mathrm{H}), 7.21(\mathrm{~m}, 2 \mathrm{H}), 7.13(\mathrm{~m}, 3 \mathrm{H}), 6.63(\mathrm{~d}, J=9.0 \mathrm{~Hz}, 1 \mathrm{H}), 5.00(\mathrm{~d}, J$ $=6.5,1 \mathrm{H}), 4.68(\mathrm{~d}, J=15.5 \mathrm{~Hz}, 1 \mathrm{H}), 4.44(\mathrm{~d}, J=15.5 \mathrm{~Hz}, 1 \mathrm{H}), 3.46(\mathrm{~m}, 2 \mathrm{H}), 3.35(\mathrm{~m}, 1 \mathrm{H}), 3.10$ $\left(\mathrm{dd}, J_{l}=15.0 \mathrm{~Hz}, J_{2}=9.0 \mathrm{~Hz}, 1 \mathrm{H}\right), 2.89\left(\mathrm{dd}, J_{l}=13.7 \mathrm{~Hz}, J_{2}=3.0 \mathrm{~Hz}, 1 \mathrm{H}\right), 2,42\left(\mathrm{dd}, J_{l}=13.5 \mathrm{~Hz}\right.$, $\left.J_{2}=11.0 \mathrm{~Hz}, 1 \mathrm{H}\right), 1.20(\mathrm{~s}, 9 \mathrm{H}) .{ }^{13} \mathrm{C}$ NMR $\left(100 \mathrm{MHz}, \mathrm{DMSO}-\mathrm{d}_{6}\right): \delta 155.2,149.5,146.1,139.3$, $136.1,129.0,128.5,128.4,128.1,127.8,127.5,125.6,124.3,77.5,71.2,54.9,50.9,50.2,35.2$, 28.1 .

(1S,2R)-\{1-Benzyl-2-hydroxy-3-[N-benzyl-(4-methoxybenzenesulfonyl)-amino]-propyl\}carbamic acid tert-butyl ester (5b). Compound $\mathbf{5 b}$ was obtained as white solid $\left(\mathrm{CH}_{2} \mathrm{Cl}_{2} / \mathrm{EtOAc}\right.$ $95 / 5)$, yield $85 \%$. $[\alpha]_{D}^{20}=+5.4^{\circ}\left(\mathrm{c}: 1.0, \mathrm{CHCl}_{3}\right) .{ }^{1} \mathrm{H}$ NMR $\left(400 \mathrm{MHz}, \mathrm{DMSO}-\mathrm{d}_{6}\right): \delta 7.78(\mathrm{~d}, J=$ $8.5 \mathrm{~Hz}, 2 \mathrm{H}), 7.28(\mathrm{~m}, 8 \mathrm{H}), 7.16-7.08(\mathrm{~m}, 4 \mathrm{H}), 6.60(\mathrm{~d}, J=8.8 \mathrm{~Hz}, 1 \mathrm{H}), 4.94(\mathrm{~d}, J=6.0 \mathrm{~Hz}, 1 \mathrm{H})$, $4.50(\mathrm{~d}, J=15.6 \mathrm{~Hz}, 1 \mathrm{H}), 4.37(\mathrm{~d}, J=15.6,1 \mathrm{H}), 3.85(\mathrm{~s}, 3 \mathrm{H}), 3.48(\mathrm{~m}, 2 \mathrm{H}), 3.35(\mathrm{~m}, 1 \mathrm{H}), 2.93(\mathrm{~m}$, 2H), $2.45(\mathrm{dd}, J=13.8 \mathrm{~Hz}, J=10.4 \mathrm{~Hz}, 1 \mathrm{H}), 1.22(\mathrm{~s}, 9 \mathrm{H}) .{ }^{13} \mathrm{C}$ NMR (100 MHz, DMSO-d 6$): \delta$ 155.2, 139.5, 136.6, 131.6, 129.2, 129.1, 128.2, 128.0, 127.8, 127.2, 125.6, 114.3, 77.4, 72.0, 54.9, 55.6, 54.8, 51.4, 50.7, 35.2, 28.1 .

(1S,2R)-\{1-Benzyl-2-hydroxy-3-[N-Benzyl-(3,4-dimethoxybenzenesulfonyl)-amino]-propyl\}carbamic acid tert-butyl ester (5c). Compound $5 \mathbf{c}$ was obtained as a white solid $\left(\mathrm{CH}_{2} \mathrm{Cl}_{2} / \mathrm{EtOAc}\right.$ 95/5) , yield $90 \%$. $[\alpha]_{D}^{20}=+6.7^{\circ}\left(\mathrm{c}: 1.0, \mathrm{CHCl}_{3}\right) .{ }^{1} \mathrm{H}$ NMR (400 MHz, DMSO-d 6$): \delta 7.45$ (dd, $J=$ 
$2.0 \mathrm{~Hz}, J=6.8 \mathrm{MHz}, 1 \mathrm{H}), 7.54(\mathrm{~m}, 10 \mathrm{H}), 6.94(\mathrm{~d}, J=8 \mathrm{~Hz}, 1 \mathrm{H}), 4.45(\mathrm{~d}, J=14,1 \mathrm{H}), 4.16(\mathrm{~d}, J=$ $27.2 \mathrm{~Hz}, 1 \mathrm{H}), 3.95(\mathrm{~s}, 3 \mathrm{H}), 3.89$ (s, 3H), $3.64(\mathrm{~d}, 1 \mathrm{H}), 3.34(\mathrm{~d}, 1 \mathrm{H}), 3.18(\mathrm{~d}, 1 \mathrm{H}), 2.8(\mathrm{~m}, 2 \mathrm{H}), 1.32$ (s, 9H). ${ }^{13} \mathrm{C}$ NMR (100 MHz, DMSO-d 6 ): $\delta$ 155.2, 149.5, 146.1, 139.3, 136.1, 129.0, 128.5, 128.4, $128.1,127.8,127.5,125.6,124.3,77.5,71.2,54.9,50.9,50.2,35.2,28.1$.

General procedure for the preparation of $N$-((2R,3S)-3-amino-2-hydroxy-4-phenylbutyl)- $N$ benzyl-R-benzenesulfonamide (6). To a stirred solution of $\mathbf{5 a - c}(0.78 \mathrm{mmol})$ in anhydrous $\mathrm{CH}_{2} \mathrm{Cl}_{2}$ $(30 \mathrm{~mL})$, trifluoroacetic acid $(13 \mathrm{~mL})$ was added at room temperature. After 1 hour the reaction mixture was concentrated and treated with toluene $(3 \times 20 \mathrm{~mL})$, evaporated in vacuum. The crude was treated with $\mathrm{Et}_{3} \mathrm{~N}$ and purified by chromatography on silica gel $\left(\mathrm{CHCl}_{3} / \mathrm{AcOEt}\right.$ 9/1).

$N$-((2R,3S)-3-Amino-2-hydroxy-4-phenylbutyl)- $N$-benzyl-4-nitrobenzenesulfonamide

(6a).

Compound was obtained as a white solid $\left(\mathrm{CH}_{2} \mathrm{Cl}_{2} / \mathrm{EtOAc} 9 / 1\right)$, yield $41 \%$. $[\alpha]_{D}^{20}=+6.4^{\circ}$ (c : 1.0 , $\left.\mathrm{CHCl}_{3}\right) .{ }^{1} \mathrm{H}$ NMR $\left(400 \mathrm{MHz}, \mathrm{DMSO}-\mathrm{d}_{6}\right): \delta 8.34(\mathrm{~d}, J=8.6 \mathrm{~Hz}, 2 \mathrm{H}), 8,11(\mathrm{bs}, 2 \mathrm{H}),$,8.06 (d, $J=8.6$ $\mathrm{Hz}, 2 \mathrm{H}), 7.26(\mathrm{~m}, 10 \mathrm{H}), 5.67(\mathrm{~d}, J=5.6,1 \mathrm{H}), 4.56(\mathrm{~d}, J=16.0 \mathrm{~Hz}, 1 \mathrm{H}), 4.49(\mathrm{~d}, J=16.0 \mathrm{~Hz}, 1 \mathrm{H})$, $3.96(\mathrm{bs}, 1 \mathrm{H}), 3.38$ (m, 2H), $3.16(\mathrm{dd}, J=14.8 \mathrm{~Hz}, J=8.8 \mathrm{~Hz}, 1 \mathrm{H}), 2.87$ (dd, $J=14.4 \mathrm{~Hz}, J=7.2 \mathrm{~Hz}$, $1 \mathrm{H}), 2.82(\mathrm{dd}, J=14.2 \mathrm{~Hz}, J=7.6 \mathrm{~Hz}, 1 \mathrm{H}) .{ }^{13} \mathrm{C}$ NMR (100 MHz, DMSO-d 6 ): $\delta 149.6,145.3$, 136.4 , 135.9, 129.3, 128.6, 128.4, 128.1, 127.6, 126.8, 124.4, 67.8, 55.2, 51.5, 49.1, 32.8 .

$N$-((2R,3S)-3-Amino-2-hydroxy-4-phenyl-butyl)- $N$-benzyl-4-methoxybenzenesulfonamide (6b). Compound was obtained as a white solid $\left(\mathrm{CH}_{2} \mathrm{Cl}_{2} / \mathrm{EtOAc} 9 / 1\right)$, yield $35 \%$. $[\alpha]_{D}^{20}=+7.5^{\circ}$ (c : 1.0 , $\left.\mathrm{CHCl}_{3}\right) .{ }^{1} \mathrm{H}$ NMR (400 MHz, DMSO-d $): \delta 7.75(\mathrm{~d}, J=8.4 \mathrm{~Hz}, 2 \mathrm{H}), 7.20(\mathrm{~m}, 10 \mathrm{H}), 6.99(\mathrm{~d}, J=8.8$ $\mathrm{Hz}, 2 \mathrm{H}), 4.33(\mathrm{~d}, J=14.4 \mathrm{~Hz}, 1 \mathrm{H}), 4.17$ (d, $J=14.4 \mathrm{~Hz}, 1 \mathrm{H}), 4.00(\mathrm{~m}, 1 \mathrm{H}), 3.88$ (s, 3H), 3.50 (m, 2H), $3.45(\mathrm{~m}, 2 \mathrm{H}), 2.79(\mathrm{~m}, 2 \mathrm{H}) .{ }^{13} \mathrm{C}$ NMR (100 MHz, DMSO-d 6$): \delta 136.5,130.8,129.3,128.5$, $128.3,128.0,127.3,126.7,114.4,68.3,56.0,55.7,51.8,49.5,32.5$.

\section{$N$-((2R,3S)-3-Amino-2-hydroxy-4-phenyl-butyl)- $N$-benzyl-3,4-methoxybenzenesulfonamide}

(6c). Compound was obtained as a white solid $\left(\mathrm{CH}_{2} \mathrm{Cl}_{2} / \mathrm{EtOAc} 9 / 1\right.$ ), yield $52 \%$. $[\alpha]_{D}^{20}=+8.3^{\circ}$ (c : 1.0, $\left.\mathrm{CHCl}_{3}\right) .{ }^{1} \mathrm{H}$ NMR (400 MHz, DMSO-d 6 ): $\delta 7.41(\mathrm{dd}, J=8 \mathrm{~Hz}, J=2.1 \mathrm{~Hz}, 1 \mathrm{H}), 7.15(\mathrm{~m}, 10 \mathrm{H})$, $6.9(\mathrm{~d}, J=8.4 \mathrm{~Hz}, 2 \mathrm{H}), 4.35$ (d, $J=14.8 \mathrm{~Hz}, 2 \mathrm{H}), 4.18$ (d, $J=14.8 \mathrm{~Hz}, 2 \mathrm{H}), 3.98$ (s, 1H), 3.92 (s, $3 \mathrm{H}), 3.82(\mathrm{~s}, 3 \mathrm{H}), 3.56(\mathrm{~s}, 1 \mathrm{H}), 3.25(\mathrm{~m}, 2 \mathrm{H}), 2.82(\mathrm{~m}, 2 \mathrm{H}) .{ }^{13} \mathrm{C}$ NMR (100 MHz, DMSO-d 6$): \delta$ $135.9,130.5,128.7,128.2$, 127.9, 127.6, 126.9, 126.5, 113.5, 67.9, 56.2, 55.8, 55.3, 51.1, 48.9, 31.8.

General procedure for the preparation of $N$-((2S,3R)-3-hydroxy-4-( $N$-benzylarylsulfonamido)-1-phenylbutan-2-yl)heteroarene-5-carboxamide $(7,8,9)$. To a solution of 5heterobenzoic acid $(0.13 \mathrm{mmol})$, EDCI $(0.20 \mathrm{mmol}), \mathrm{HOBt}(0.20 \mathrm{mmol})$ in anhydrous $\mathrm{CH}_{2} \mathrm{Cl}_{2}$, a solution of amine 6a-c $(0.13 \mathrm{mmol})$ and diisopropylethylamine $(0.78 \mathrm{mmol})$ in anhydrous $\mathrm{CH}_{2} \mathrm{Cl}_{2}$ was added at $0^{\circ} \mathrm{C}$ under argon atmosphere and it was allowed to stir for $16 \mathrm{~h}$ at room temperature. 
The reaction mixture was quenched with water and extracted with $\mathrm{CH}_{2} \mathrm{Cl}_{2}$. The organic layers were dried on $\mathrm{Na}_{2} \mathrm{SO}_{4}$, filtered and concentrated under reduced pressure. The residue was purified by silica gel column chromatography $\left(\mathrm{CH}_{2} \mathrm{Cl}_{2} / \mathrm{AcOEt} 9 / 1\right)$ tu furnish inhibitors 7a-c, 8a-c, 9a-c.

\section{$N$-((2S,3R)-3-hydroxy-4-( $N$-benzyl-4-nitrophenylsulfonamido)-1-phenylbutan-2-}

yl)benzo[b]thiophene -5-carboxamide (7a). Following the general procedure the compound 7a was obtained as a white solid, yield $50 \% .[\alpha]_{D}^{20}=+14.5^{\circ}$ (c : $\left.1, \mathrm{CHCl}_{3}\right) .{ }^{1} \mathrm{H} \mathrm{NMR}(400 \mathrm{MHz}$, $\left.\mathrm{CDCl}_{3}\right): \delta 8.29(\mathrm{~d}, J=8.8 \mathrm{~Hz}, 1 \mathrm{H}), 7.95(\mathrm{~m}, 4 \mathrm{H}), 7.54(\mathrm{~d}, J=6.4 \mathrm{~Hz}, 1 \mathrm{H}), 7.44(\mathrm{~d}, \mathrm{~J}=8.4 \mathrm{~Hz}, 1 \mathrm{H})$, $7.75(\mathrm{~m}, 12 \mathrm{H}), 6.07(\mathrm{~d}, J=8.0 \mathrm{~Hz}, 1 \mathrm{H}), 4.43(\mathrm{~m}, 2 \mathrm{H}), 4.20(\mathrm{~m}, 1 \mathrm{H}), 3.66(\mathrm{~m}, 1 \mathrm{H}), 3.36(\mathrm{~m}, 2 \mathrm{H})$, $3.98(\mathrm{~m}, 2 \mathrm{H}) .{ }^{13} \mathrm{C} \mathrm{NMR}\left(100 \mathrm{MHz}, \mathrm{CDCl}_{3}\right): \delta 168.6,150.0,144.9,143.0,139.4,137.1,135.0$, 129.8, 129.3, 129.0, 128.8, 128.7, 128.4, 123.3, 126.8, 124.3, 124.1, 122.7, 122.1, 71.7, 54.9, 53.6, $51.5,35.3$.

$N$-((2S,3R)-3-hydroxy-4-( $N$-benzyl-4-methoxyphenylsulfonamido)-1-phenylbutan-2-

yl)benzo[b]thiophene -5-carboxamide (7b). Following the general procedure the compound $\mathbf{7 b}$ was obtained as a white solid, yield $57 \% \cdot[\alpha]_{D}^{20}=+1.5^{\circ}\left(\mathrm{c}: 0.32, \mathrm{CHCl}_{3}\right) .{ }^{1} \mathrm{H}$ NMR $(400 \mathrm{MHz}$, $\left.\mathrm{CDCl}_{3}\right): \delta 7.98(\mathrm{~s}, 1 \mathrm{H}), 7.88(\mathrm{~d}, J=8.4 \mathrm{~Hz}, 1 \mathrm{H}), 7.71(\mathrm{~d}, J=9.2 \mathrm{~Hz}, 2 \mathrm{H}), 7.53(\mathrm{~d}, J=5.6 \mathrm{~Hz}, 1 \mathrm{H})$, $7.46(\mathrm{~d}, J=8.4 \mathrm{~Hz}, 1 \mathrm{H}), 7.37(\mathrm{~d}, J=5.2 \mathrm{~Hz}, 1 \mathrm{H}), 7.20(\mathrm{~m}, 10 \mathrm{H}), 6.94(\mathrm{~d}, J=9.2 \mathrm{~Hz}, 2 \mathrm{H}), 6.03(\mathrm{~d}, J$ $=8.0 \mathrm{~Hz}, 1 \mathrm{H}), 4.25(\mathrm{~m}, 3 \mathrm{H}), 3.86(\mathrm{~s}, 3 \mathrm{H}), 3.50(\mathrm{~m}, 1 \mathrm{H}), 3.34(\mathrm{~m}, 1 \mathrm{H}), 3.08(\mathrm{~m}, 2 \mathrm{H}), 3.98(\mathrm{~m}, 1 \mathrm{H})$. ${ }^{13} \mathrm{C}$ NMR (100 MHz, $\left.\mathrm{CDCl}_{3}\right): \delta 168.1,163.1,142.8,139.3,137.5,135.9,130.1,129.8,129.4$, 129.4, 128.8, 128.7, 128.5, 128.1, 127.9, 126.6, 124.1, 121.6, 122.5, 122.2, 114.4, 72.0, 55.6, 54.5, $54.2,52.5,35.2$.

\section{$N$-((2S,3R)-3-hydroxy-4-( $N$-benzyl-3,4-dimethoxyphenylsulfonamido)-1-phenylbutan-2-}

yl)benzo[b]thiophene -5-carboxamide (7c). Following the general procedure the compound 7c was obtained as a white solid, yield $55 \% .[\alpha]_{D}^{20}=+4.6^{\circ}$ (c : $0.1, \mathrm{CHCl}_{3}$ ). ${ }^{1} \mathrm{H}$ NMR (400 MHz, $\left.\mathrm{CDCl}_{3}\right): \delta 7.99$ (d, $\left.J=1.6 \mathrm{~Hz}, 1 \mathrm{H}\right), 7.87(\mathrm{~d}, J=8.8 \mathrm{~Hz}, 1 \mathrm{H}), 7.45(\mathrm{~m}, 15 \mathrm{H}), 6.88(\mathrm{~d}, J=8.4 \mathrm{~Hz}, 1 \mathrm{H})$, $6.09(\mathrm{~d}, J=8.4 \mathrm{~Hz}, 1 \mathrm{H}), 4.13(\mathrm{~m}, 3 \mathrm{H}), 3.98(\mathrm{~s}, 3 \mathrm{H}), 3.79$ (s, 3H), $3.58(\mathrm{~m}, 1 \mathrm{H}), 3.38(\mathrm{~m}, 1 \mathrm{H}), 3.06$ (m, 2H), $2.98(\mathrm{~m}, 1 \mathrm{H}) .{ }^{13} \mathrm{C}$ NMR $\left(100 \mathrm{MHz}, \mathrm{CDCl}_{3}\right): \delta 168.1,152.8,149.2,142.8,139.3,137.5$, $135.9,130.0,129.4,128.8,128.7,128.6,128.1,128.0,126.6,124.2,122.6,122.2,121.2,110.7$, 109.6, 72.0, 56.2, 56.1, 54.4, 54.3, 52.3, 35.2.

\section{$N$-((2S,3R)-3-hydroxy-4-( $N$-benzyl-4-nitrophenylsulfonamido)-1-phenylbutan-2-yl)benzofuran}

-5-carboxamide (8a). Following the general procedure the compound 8a was obtained as a white solid, yield 53\%. $[\alpha]_{D}^{20}=+3.1^{\circ}\left(\mathrm{c}: 0.22, \mathrm{CHCl}_{3}\right) .{ }^{1} \mathrm{H} \mathrm{NMR}\left(400 \mathrm{MHz}, \mathrm{CDCl}_{3}\right): \delta 8.18(\mathrm{~d}, J=8.8$ $\mathrm{Hz}, 2 \mathrm{H}), 7.98(\mathrm{~d}, J=8.8 \mathrm{~Hz}, 2 \mathrm{H}), 8.80(\mathrm{~s}, 1 \mathrm{H}), 8.70(\mathrm{~s}, 1 \mathrm{H}), 7.45(\mathrm{~m}, 12 \mathrm{H}), 6.82(\mathrm{~s}, 1 \mathrm{H}), 6.03(\mathrm{~d}, J$ $=8.0 \mathrm{~Hz}, 1 \mathrm{H}), 4.25(\mathrm{~m}, 2 \mathrm{H}), 4.16(\mathrm{~m}, 1 \mathrm{H}), 3.67(\mathrm{~m}, 1 \mathrm{H}), 3.17(\mathrm{~m}, 2 \mathrm{H}), 3.01(\mathrm{~m}, 2 \mathrm{H}) .{ }^{13} \mathrm{C} \mathrm{NMR}$ 
$\left(100 \mathrm{MHz}, \mathrm{CDCl}_{3}\right): \delta 168.6,156.7,150.0,146.5,137.1,135.0,129.2,128.8,128.7,128.4,128.3$, 127.6, 126.8, 124.3, 123.1, 120.7, 111.5, 71.7, 55.0, 53.5, 51.5, 35.3.

\section{$N$-((2S,3R)-3-hydroxy-4-( $N$-benzyl-4-methoxyphenylsulfonamido)-1-phenylbutan-2-}

yl)benzofuran -5-carboxamide (8b). Following the general procedure the compound $\mathbf{8 b}$ was obtained as a white solid, yield 54\%. $[\alpha]_{D}^{20}=+3.6^{\circ}$ (c : $\left.1, \mathrm{CHCl}_{3}\right) .{ }^{1} \mathrm{H} \mathrm{NMR}\left(400 \mathrm{MHz}, \mathrm{CDCl}_{3}\right): \delta$ $7.79(\mathrm{~s}, 1 \mathrm{H}), 7.70(\mathrm{~m}, 1 \mathrm{H}), 7.47(\mathrm{~m}, 2 \mathrm{H}), 7.18(\mathrm{~m}, 10 \mathrm{H}) ; 6.95(\mathrm{~d}, J=8.4 \mathrm{~Hz}, 2 \mathrm{H}), 6.81(\mathrm{~m}, 1 \mathrm{H})$, $6.00(\mathrm{~d}, J=8 \mathrm{~Hz}, 1 \mathrm{H}), 4.25(\mathrm{~m}, 3 \mathrm{H}), 3.87(\mathrm{~s}, 3 \mathrm{H}), 3.6(\mathrm{~m}, 1 \mathrm{H}), 3.55(\mathrm{~m}, 1 \mathrm{H}), 3.35(\mathrm{~m}, 1 \mathrm{H}), 3.18(\mathrm{~m}$, 2H), $2.98(\mathrm{~m}, 1 \mathrm{H}) .{ }^{13} \mathrm{C} \mathrm{NMR}\left(100 \mathrm{MHz}, \mathrm{CDCl}_{3}\right): \delta 168.2,163.1,156.6,146.4,137.5,135.9,129.4$, $129.4,129.3,128.8,128.6,128.5,128.0,127.5,126.5,123.2,120.6,114.6,114.4,111.4,106.9$, $72.6,55.6,54.5,54.2,52.5,35.2,31.9$.

\section{$N$-((2S,3R)-3-hydroxy-4-( $N$-benzyl-3,4-dimethoxyphenylsulfonamido)-1-phenylbutan-2-}

yl)benzofuran -5-carboxamide (8c). Following the general procedure the compound 8c was obtained as a white solid, yield $56 \%$. $[\alpha]_{D}^{20}=+19.5^{\circ}\left(\mathrm{c}: 1, \mathrm{CHCl}_{3}\right) .{ }^{1} \mathrm{H} \mathrm{NMR}\left(400 \mathrm{MHz}, \mathrm{CDCl}_{3}\right): \delta$ $7.80(\mathrm{~s}, 1 \mathrm{H}), 7.6(\mathrm{~d}, J=2.0 \mathrm{~Hz}, 1 \mathrm{H}), 7.43(\mathrm{~m}, 3 \mathrm{H}), 7.20(\mathrm{~m}, 12 \mathrm{H}), 6.89(\mathrm{~d}, J=8.4 \mathrm{~Hz}, 1 \mathrm{H}), 6.8(\mathrm{~d}, J$ $=2.1 \mathrm{~Hz}, 1 \mathrm{H}), 6.1(\mathrm{~d}, J=7.6 \mathrm{~Hz}, 1 \mathrm{H}), 3.93(\mathrm{~s}, 3 \mathrm{H}), 3.80(\mathrm{~s}, 3 \mathrm{H}), 3.52(\mathrm{~m}, 1 \mathrm{H}), 3.37(\mathrm{~m}, 1 \mathrm{H}), 3.06$ (m, 2H), $2.92(\mathrm{~m}, 1 \mathrm{H}) .{ }^{13} \mathrm{C}$ NMR $\left(100 \mathrm{MHz}, \mathrm{CDCl}_{3}\right): \delta 168.2,156.6,152.8,130.1,129.4,128.9$, $128.8,128.7,128.5,128.1,127.5,126.6,123.2$, 121.1, 120.7, 111.6, 110.7, 109.6, 106.9, 72.0, 56.2, $56.1,54.3,52.4,35.2,30.9$.

$N$-((2S,3R)-3-hydroxy-4-(N-benzyl-4-nitrophenylsulfonamido)-1-phenylbutan-2-yl)1H-indol -

5-carboxamide (9a). Following the general procedure the compound 9a was obtained as a white solid, yield 33\%. $[\alpha]_{D}^{20}=+25.8^{\circ}\left(\mathrm{c}: 1.3, \mathrm{CHCl}_{3}\right) .{ }^{1} \mathrm{H}$ NMR $\left(400 \mathrm{MHz}, \mathrm{CDCl}_{3}\right): \delta 8.64(\mathrm{~s}, 1 \mathrm{H}), 7.84$ (s, 1H), $7.72(\mathrm{~d}, J=8.8 \mathrm{~Hz}, 2 \mathrm{H}), 7.40-7.15(\mathrm{~m}, 14 \mathrm{H}), 6.93(\mathrm{~d}, J=8.8 \mathrm{~Hz}, 2 \mathrm{H}), 6.59(\mathrm{~s}, 1 \mathrm{H}), 6.03$ (d, $J=6 \mathrm{~Hz}, 1 \mathrm{H}), 4.25(\mathrm{~m}, 2 \mathrm{H}), 3.84(\mathrm{~s}, 3 \mathrm{H}), 3.50(\mathrm{~m}, 1 \mathrm{H}), 3.35(\mathrm{dd}, J=15 \mathrm{~Hz}, J=4.4 \mathrm{~Hz}, 1 \mathrm{H}), 3.17$ (m, 2H), $2.90(\mathrm{~m}, 2 \mathrm{H}) .{ }^{13} \mathrm{C}$ NMR $\left(100 \mathrm{MHz}, \mathrm{CDCl}_{3}\right): \delta 169.2,163.0,137.7,136.6,136.0,129.5$, 129.4, 128.8, 128.7, 128.5, 128.0, 127.4, 126.5, 125.7, 120.8, 120.4, 114.4, 111.0, 103.6, 72.1, 55.6, $54.5,54.2,52.5,35.3$

$N$-((2S,3R)-3-hydroxy-4-( $N$-benzyl-4-methoxyphenylsulfonamido)-1-phenylbutan-2-yl)1H-

indol -5-carboxamide (9b). Following the general procedure the compound $\mathbf{9 b}$ was obtained as a white solid, yield 43\%. $[\alpha]_{D}^{20}=+10.2^{\circ}\left(\mathrm{c}: 0.4, \mathrm{CHCl}_{3}\right) .{ }^{1} \mathrm{H} \mathrm{NMR}\left(400 \mathrm{MHz}, \mathrm{CDCl}_{3}\right): \delta 8.47(\mathrm{~s}$, 1H) $8.24(\mathrm{~d}, J=8.8 \mathrm{~Hz}, 2 \mathrm{H}), 7.90(\mathrm{~d}, J=8.8 \mathrm{~Hz}, 2 \mathrm{H}), 7.81(\mathrm{~s}, 1 \mathrm{H}), 7.37-7.17(\mathrm{~m}, 14 \mathrm{H}), 6.59(\mathrm{~s}$, $1 \mathrm{H}), 6.04(\mathrm{~d}, J=7.2 \mathrm{~Hz}, 1 \mathrm{H}), 4.52$ (d, $J=14.4 \mathrm{~Hz}, 1 \mathrm{H}), 4.37$ (d, $J=14.4 \mathrm{~Hz}, 1 \mathrm{H}), 4.18$ (m, 2H), 3.81-3.24 (m, 2H), 2.95 (m, 2H). ${ }^{13} \mathrm{C}$ NMR (100 MHz, $\left.\mathrm{CDCl}_{3}\right)$ : 169.7, 149.9, 145.0, 137.7, 137.2, $135.1,129.3,128.8,128.7,128.6,128.4,128.3,127.5,126.8,125.8,125.3,124.3,120.9,120.4$, $111.1,103.7,71.8,55.1,53.4,51.3,35.6$. 
$N$-((2S,3R)-3-hydroxy-4-( $N$-benzyl-3,4-dimethoxyphenylsulfonamido)-1-phenylbutan-2-yl)1Hindol-5-carboxamide (9c). Following the general procedure the compound 9c was obtained as a white solid, yield 44\%. $[\alpha]_{D}^{20}=+18.7^{\circ}$ (c : $1, \mathrm{CHCl}_{3}$ ). ${ }^{1} \mathrm{H} \mathrm{NMR}\left(400 \mathrm{MHz}, \mathrm{CDCl}_{3}\right.$ ): $\delta 8.76$ (bs, 1H), $7.85(\mathrm{~s}, 1 \mathrm{H}), 7.37(\mathrm{~m}, 3 \mathrm{H}), 7.23(\mathrm{~m}, 11 \mathrm{H}), 6.85(\mathrm{~d}, J=8.4 \mathrm{~Hz}, 1 \mathrm{H}), 6.56(\mathrm{~s}, 1 \mathrm{H}), 6.12(\mathrm{~d}, J=$ $8 \mathrm{~Hz}, 1 \mathrm{H}), 4.37(\mathrm{~d}, J=14.8 \mathrm{~Hz}, 1 \mathrm{H}), 3.90(\mathrm{~s}, 3 \mathrm{H}), 3.76(\mathrm{~s}, 3 \mathrm{H}), 3.55(\mathrm{~m}, 1 \mathrm{H}), 3.40(\mathrm{dd}, J=12 \mathrm{~Hz}, J=$ $4 \mathrm{~Hz}, 1 \mathrm{H}), 3.10(\mathrm{~m}, 2 \mathrm{H}), 2.92(\mathrm{~m}, 1 \mathrm{H}) .{ }^{13} \mathrm{C} \mathrm{NMR}\left(100 \mathrm{MHz}, \mathrm{CDCl}_{3}\right): \delta 169.2,152.6,149.1,137.6$, $135.8,130.1,129.3,128.7,128.6,128.4,127.9,127.7,127.4,126.4,125.7,125.3,121.1,120.7$, $120.2,110.9,110.6,109.6,103.4,72.0,60.3,56.1,55.9,54.4,54.1,52.3,35.2,31.4,29.5,22.5$, 20.9, 14.0 .

(2R,3S)-3-amino-1-(benzylamino)-4-phenylbutan-2-ol (10). A solution of $(2 S, 3 S)$-1,2-epoxy-3(Boc-amino)-4-phenylbutane $3(1.6 \mathrm{mmol})$ and benzylamine $(1.5 \mathrm{mmol})$ in $i$-PrOH $(10 \mathrm{~mL})$ was stirred under reflux for $16 \mathrm{~h}$. The reaction mixture was rotary evaporated, and the crude product was purified by recrystallization in methanol/water (7:3) to afford compound $\mathbf{4}$ as a white solid. Then product 4 ( $1 \mathrm{mmol})$ was then dissolved in $\mathrm{MeCN}(10 \mathrm{ml})$ and tosic acid monohydrate was added (3 $\mathrm{mmol}$ ); the resulting mixture was stirred at room temperature for $5 \mathrm{~h}$. The precipitate formed was filtered off and washed with $\mathrm{Et}_{2} \mathrm{O}$ to give $\mathbf{1 0}$ as a white solid, $60 \%$ yield. ${ }^{1} \mathrm{H}$ NMR (400 MHz, DMSO-d 6 ): $\delta 9.00$ (bs, 1H), 8.89 (bs, 1H), 7.97 (bs, 3H), 7.49 (d, J = $7.6 \mathrm{~Hz}, 4 \mathrm{H}), 7.45$ (m, 5H), $7.30(\mathrm{~m}, 5 \mathrm{H}), 7.13(\mathrm{~d}, J=7.6 \mathrm{~Hz}, 4 \mathrm{H}), 6.11(\mathrm{bs}, 1 \mathrm{H}), 4.15(\mathrm{~m}, 2 \mathrm{H}), 4.06(\mathrm{~d}, J=10.4 \mathrm{~Hz}, 1 \mathrm{H}), 3.53$ (m, 1H), 3.13 (m, 1H), 2.86 (m, 3H), 2.29 (s, 6H). ${ }^{13} \mathrm{C}$ NMR (100 MHz, DMSO-d $)$ : $\delta 145.3,137.9$, $135.9,131.3$, 130.2, 129.3, 129.1, 128.9, 128.7, 128.1, 127.0, 125.5, 65.7, 54.9, 50.2, 47.3, 33.1, 20.8 .

\subsection{In vitro activity test}

$\mathrm{IC}_{50}$ values were determined at $\mathrm{pH} 5.5$ using recombinant wild-type HIV-1 PR from Bachem and the fluorogenic substrate Abz-Thr-Ile-Nle-Phe(p-NO2)-Gln-Arg-NH2 (Abz-NF/-6; Bachem AG, Bubendorf, $\mathrm{CH}$ ). Darunavir was used in this assay as reference inhibitor for titration of the active enzyme.

\subsection{Molecular modeling}

A crystallographic structure of the wt-HIV-Pr complex with Darunavir (PDB id. 4LL3) was used as the starting geometry of the model complexes. The structure was prepared by adding hydrogen atoms, removing water crystallization molecules but keeping the essential one inside the catalytic site, and choosing always the most symmetrical option for aminoacid side chains allowing more solutions. The structure was then optimized with the Amber* force field as implemented in the Schrödinger suite [26, 27]. After docking, the complexes were thermalized by a MD run carried out with Yasara (NTV, $300{ }^{\circ} \mathrm{K}, 500 \mathrm{ps}$ ) and finally optimized as previously described. The models of 
the heterocylcles - methane complexes were obtained at the MO62X/6-311++G(d,p) level with Gaussian 09 [28].

\section{Results and Discussion}

\subsection{Chemistry}

The preparation of aromatic sulfonamides (general structure A, figure 2) started from homochiral $\mathrm{N}$ Boc protected amino epoxide 3, keeping the established stereochemistry during the synthesis [29, 30]. The epoxide was firstly opened with benzylamine to afford the monoprotected diaminoalcohol 4. Then, the substituted benzenesulfonyl groups were introduced and the $N$-Boc group efficiently displaced by treatment with trifluoroacetic acid in dichloromethane. The crude ammonium trifluoroacetate derivatives were treated with $\mathrm{NEt}_{3}$, affording the free amines 6a-c. Amines were reacted with 5-heteroarylcarboxylic acids, previously activated with 1-ethyl-3-(3dimethylaminopropyl)carbodiimide and hydroxybenzotriazole. Thus, final products $7 \mathbf{a}-\mathbf{c}, \mathbf{8 a - c}$ and 9a-c were obtained in four steps and excellent overall yield (scheme 1).<smiles>COc1ccc(C(C)(C)C)cc1</smiles><smiles>[X]c1ccc(C(=O)N[C@@H](Cc2ccccc2)[C@H](O)CN(Cc2ccccc2)S(=O)(=O)c2ccccc2)cc1</smiles>

$X=S, 7 a-c$

$X=0,8 a-c$

$\mathrm{X}=\mathrm{NH}, \mathbf{9 a}-\mathrm{c}$<smiles>[R]c1ccc(S(=O)(=O)N(Cc2ccccc2)C[C@H](O)[C@@H](N)Cc2ccccc2)cc1</smiles>

$6 a-c$

Scheme 1. Synthesis of inhibitors 7a-c, 8a-c and 9a-c: (a) $\mathrm{BnNH}_{2}, i-\mathrm{PrOH}, 60^{\circ} \mathrm{C}, 4 \mathrm{~h}(88 \%$ yield); (b) arylsulfonyl chloride, $\mathrm{Et}_{3} \mathrm{~N}, \mathrm{CH}_{2} \mathrm{Cl}_{2}$, rt, 24h (5a 94\%; 5b 85\%; 5c 90\%); (c) $\mathrm{TFA} / \mathrm{CH}_{2} \mathrm{Cl}_{2} 30 \%$, $\mathrm{rt}, 1 \mathrm{~h}$; then $\mathrm{Et}_{3} \mathrm{~N}$; (d) 5-heteroarylcarboxylic acid, EDC, $\mathrm{HOBt}$, then 6a-c, $i \mathrm{Pr}_{2} \mathrm{NEt}, \mathrm{CH}_{2} \mathrm{Cl}_{2}, 24 \mathrm{~h}, \mathrm{rt}$ (7a, 50\%; 7b 57\%; 7c 55\%; 8a 53\%; 8b 54\%; 8c 56\%; 9a 33\%; 9b 43\%; 9c 44\%). 
This synthetic pathway appears very solid, high yielding and general, irrespective on the $N$-group, the sulfonamide or the type of heteroaryl moiety chosen. The easy access of substrates represents an open door to molecules with sinergic biological activity, as anticancer activity, especially because there has been growing interest in repurposing PIs for the treatment of cancer [31].

Despite this pathway proved to be solid, diversity-oriented synthesis was studied to introduce different functionalities according to needs. In particular the removal of the Boc group immediately after opening the commercial epoxide 3 with benzylamine allowed to diamino alchol 10. In this way it should be possible to introduce firstly the desired heteroaryl moiety on the primary amine and then the different aromatic sulfonyls on the sterically hindering secondary amine. Unfortunately, this strategy proved not applicable because under these conditions diamine $\mathbf{1 0}$ did not react to afford the desired heterocarboxyamide derivative (scheme 2).

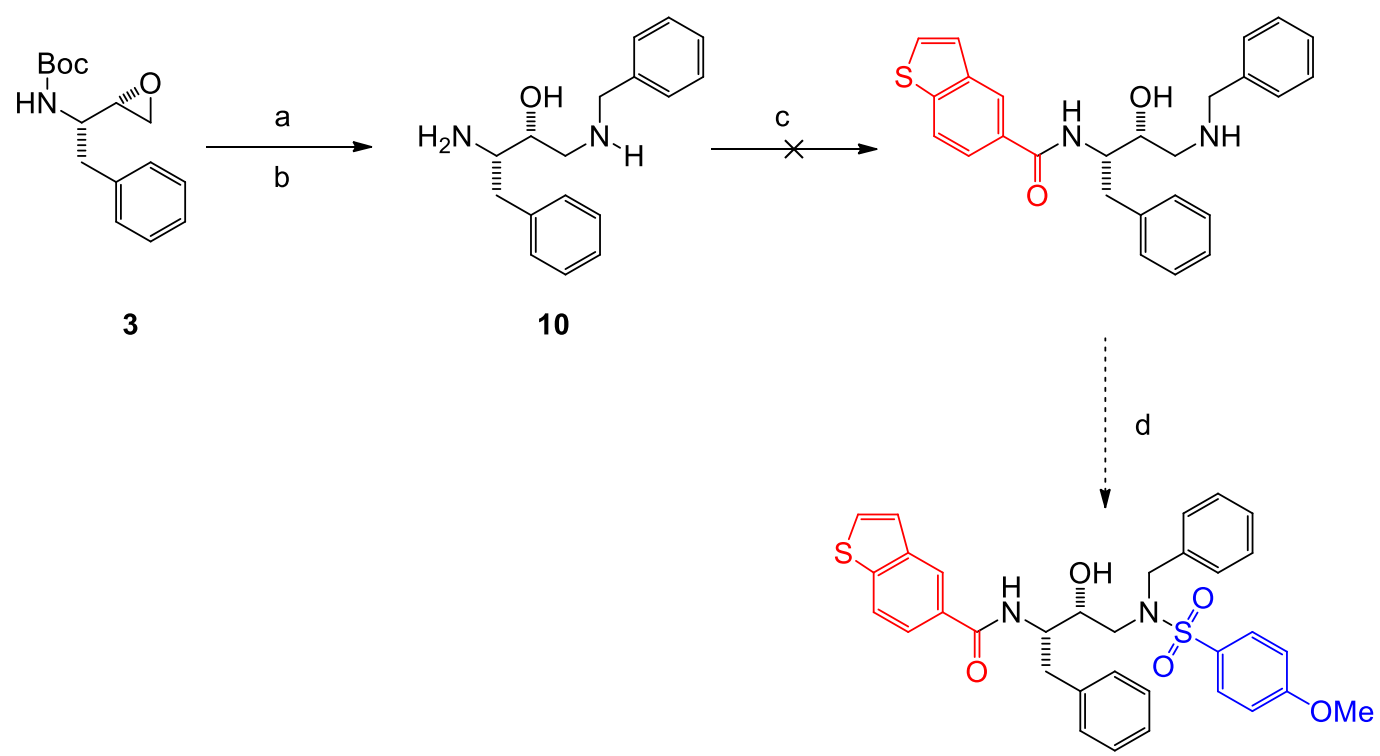

Scheme 2. (a) $\mathrm{BnNH}_{2}, i-\mathrm{PrOH}, 60^{\circ} \mathrm{C}$, 4h; (b) $p$ - $\mathrm{TsOH}, \mathrm{MeCN}, 5 \mathrm{~h}, \mathrm{rt}(60 \%$ yield from 3); (c) 5 heteroarylcarboxylic acid, EDC, $\mathrm{HOBt}$, then 10, $i \mathrm{Pr}_{2} \mathrm{NEt}, \mathrm{CH}_{2} \mathrm{Cl}_{2}, 24 \mathrm{~h}$, rt; (d) p-methoxybenzene sulfonyl chloride, $\mathrm{Et}_{3} \mathrm{~N}, \mathrm{CH}_{2} \mathrm{Cl}_{2}$.

\subsection{In vitro activity}

$\mathrm{IC}_{50}$ values were obtained on recombinant wild type HIV protease by measuring the initial rates of hydrolysis of the fluorogenic substrate Abz-Thr-Ile-Nle-Phe $\left(\mathrm{NO}_{2}\right)$-Gln-Arg (table1) [13-18]. Results are the mean of three independent experiments and are reported in table 1. 
Table 1. In vitro inhibition activity of compounds 7 .

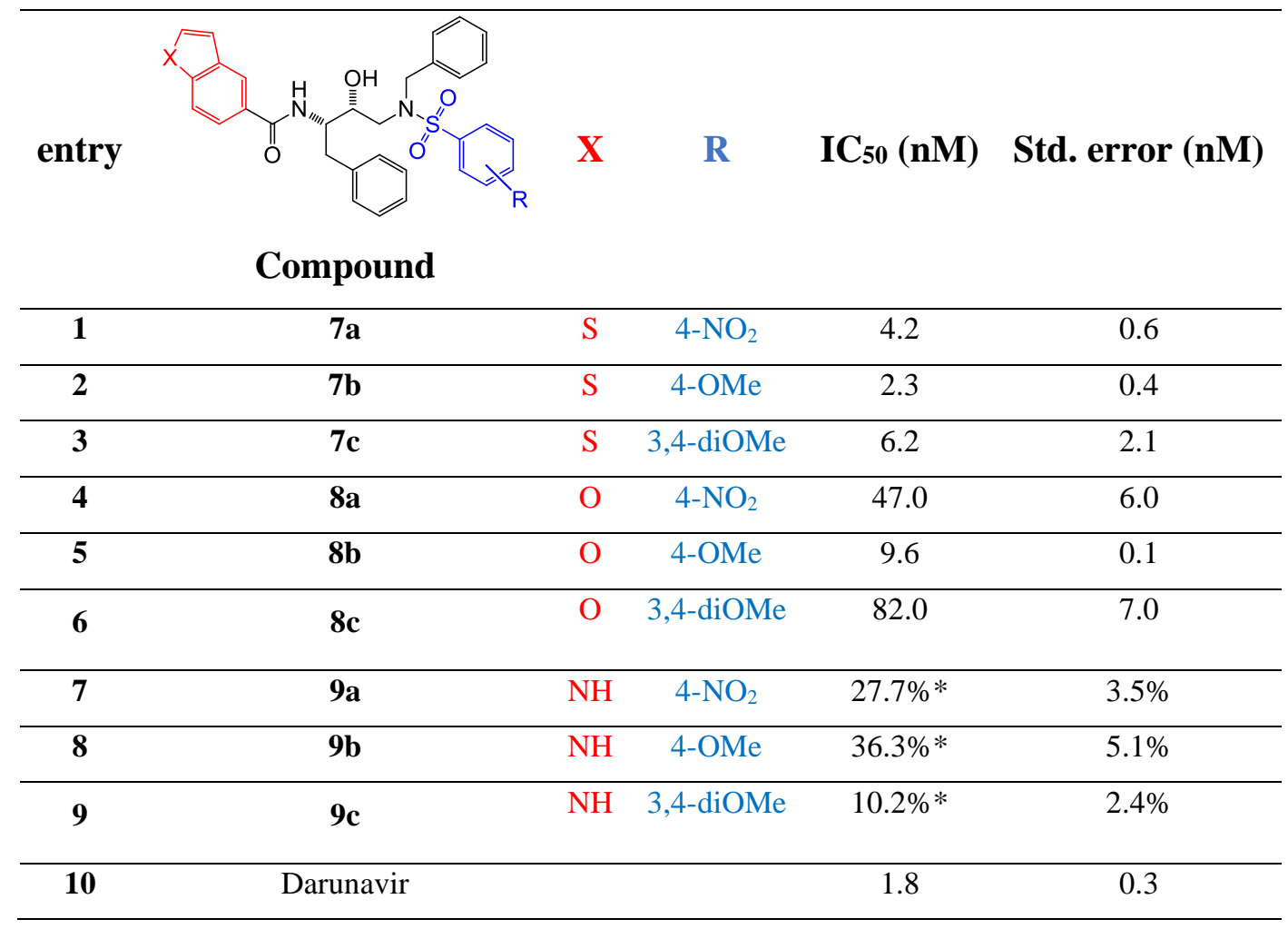

*residual activity at 500pM inhibitor.

All the inhibitors proved to be active, with generally excellent $\mathrm{IC}_{50}$ values. The indole derivatives 9a-c are the most powerful inhibitors, and perform better than darunavir under our experimental conditions. We do not report the $\mathrm{IC}_{50}$ s for the indole compounds, as their values appear less than the enzyme concentration used in the test (they can be considered minor than $0.6 \mathrm{nM}$ ). The heterocyclic system at P2 plays the major role in controlling the activity of the inhibitor: one order of magnitude in affinity is lost on changing the indole to benzothiophene and compounds 7a-c show nanomolar $\mathrm{IC}_{50}$ s. A further decrease is observed with benzofuran at $\mathrm{P} 2$, as in inhibitors 8a-c, with tenth nanomolar $\mathrm{IC}_{50} \mathrm{~s}$.

A minor effect is given by the substituents at the arylsulfonamide group at P2', where, at least in series 7 and 8, the 4-methoxyphenyl moiety seems slightly better than 4-nitrophenyl and 3,4dimethoxyphenyl. This effect, if present, cannot be evaluated in the more active compounds 9a-c.

The beneficial effect of indole in comparison with benzothiophene and benzofuran was alreaady observed in our previous studies on compounds $\mathbf{1}$ and $\mathbf{2}$, which are different at P1' (an isobutyl group is present), and in $\mathbf{2}$ also as to the lenght of the chain connecting P2 with the core (a one-atom longer carbamate linker).

\subsection{Molecular modeling}


Models were therefore built to gain insight on the structural effects at the origin of the observed activities.

The optimized complexes of HIV-Pr with all the indole derivatives 9a-c, 1c and 2c were obtained and compared with the experimental crystallographic structure of the complex of darunavir with the enzyme. The model complexes of the benzothiophene and benzofuran derivatives were then also obtained.

All the heterocyclic systems are hosted by the S2 site of the protein in a very similar way. An overlay of the structures of Darunavir and $\mathbf{9 b}$ is reported in figure 3a, while the overlay of the structures of $\mathbf{7 b}, \mathbf{8 b}, \mathbf{9 b}$ is reported in figure $3 \mathrm{~b}$.

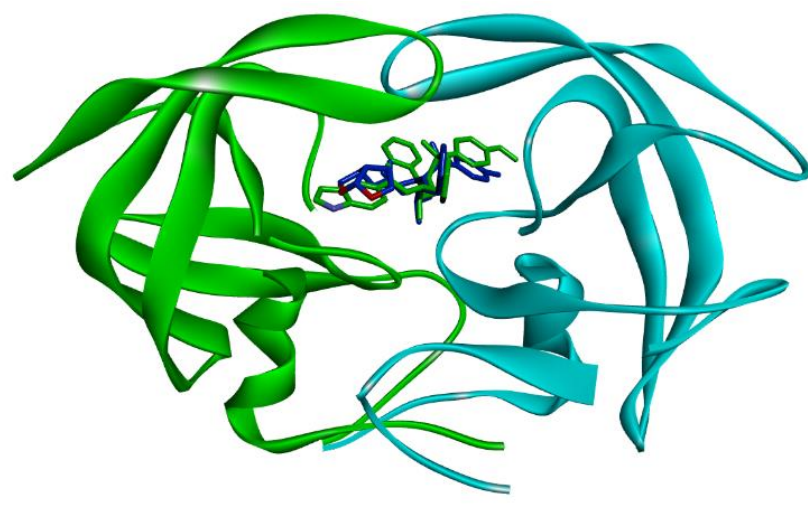

a

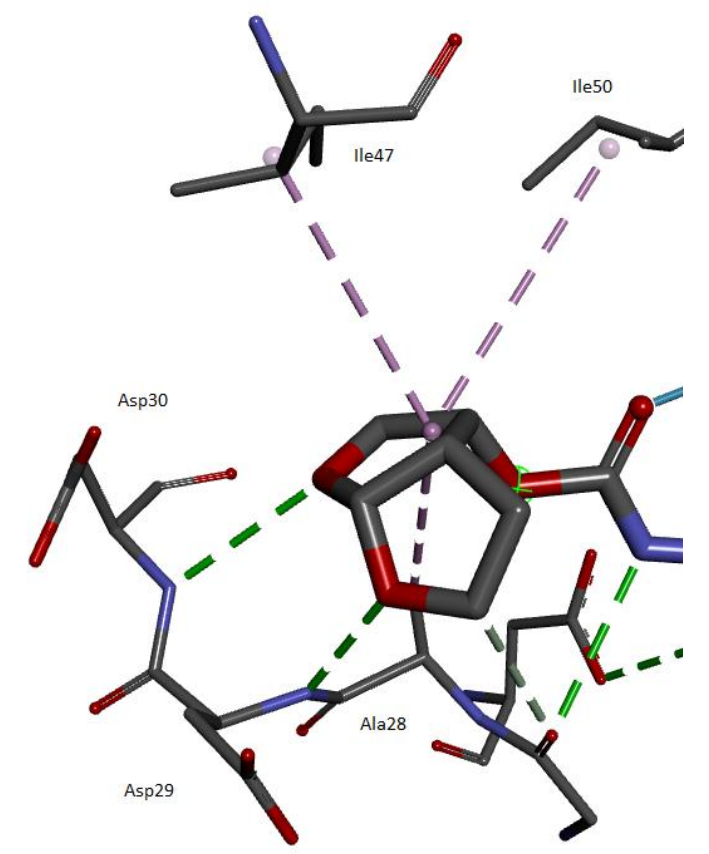

c

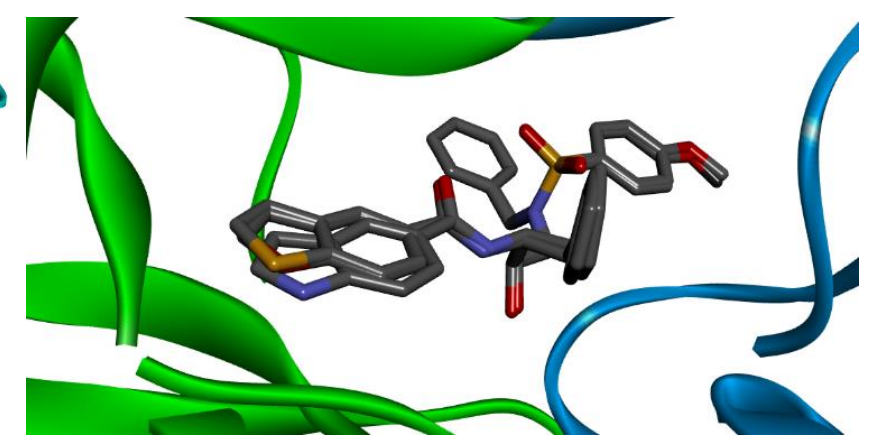

b

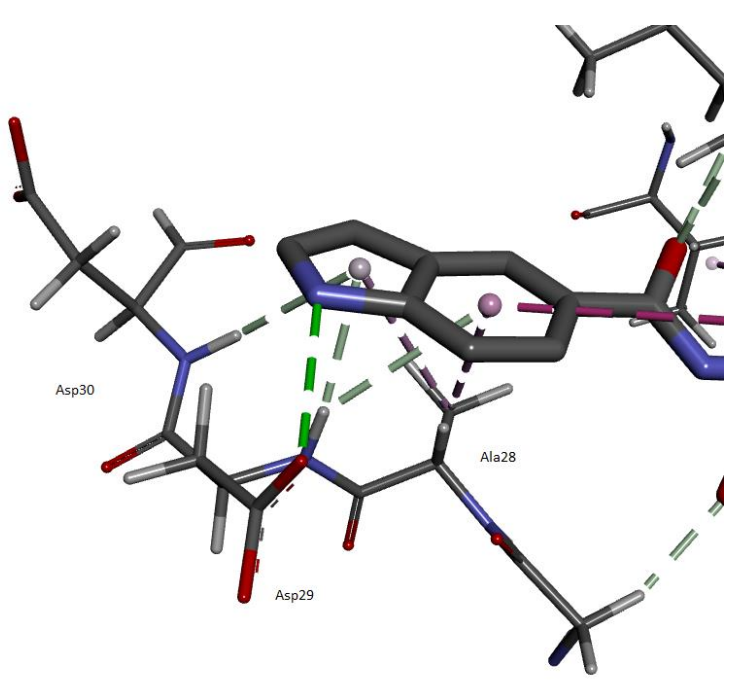

d

Figure 3. a: overlay of the crystallographic structure of the Hiv-pr complex with Darunavir and the model structure of the complex of $\mathbf{9 b}$; b: overlay of the model structures of the complexes of $\mathbf{7 b}, \mathbf{8 b}$ and $9 \mathrm{~b}$ with the protease; c: interactions of the dioxabicyclo octane group of Darunavit with the protein; $d$ : interactions of the indole system of $\mathbf{9 b}$ with the protein. 
The heteroatoms (S, O, N) are closely superimposed, while the heterocyclic systems are quite more exposed to the solvent than the dioxabicyclo octane side chain of Darunavir. Nevertheless, the indole derivatives inhibit the enzyme better than Darunavir. Details of the interactions established by Darunavir and $\mathbf{9 b}$ are reported in figures $3 \mathrm{c}$ and $3 \mathrm{~d}$.

A clear difference is given by the ability of the indole NH group to act as an hydrogen bond donor towards the carboxylate group of Asp30. This interaction can not be established by Darunavir, nor by our benzofuran - benzothiophene compounds, which can only accept hydrogen bonds. However, the heteroatoms in 7, 8 and 9 point outside the binding site, and are largely exposed to the solvent. Thus, the interaction with Asp30 is expected to be rather weak, and other effects are most likely operating. The aromatic rings of our inhibitors can clearly establish significant interactions mediated by their $\pi$ systems. A recent study has compared at different levels of theory the ability of indole, thiophene and benzofuran as partners in the formation of $\pi-\pi$ stacking interactions with DNA bases [32]. Very interestingly, indole was capable to establish the strongest $\pi-\pi$ stacking interactions, followed by benzothiophene, and then by benzofuran. This order resembles that of the inhibitory activity of our compounds. By the way, aromatic side chains are not present in subsite S2 of HIV-Pr, rather there is a number of methyl groups wallpapering the surface of S2, and those from Ala28 and Ile47 (to a minor extent) are found to interact with the heterocyclic side chains of our compounds. We have therefore carried out a preliminary evaluation on the ability of indole, thiophene and benzofuran in $\mathrm{CH}_{3} / \pi$ interactions by modelling their complexes with methane. We have followed one of the approaches reported by Toupkanloo and Rahmani, optimizing the structures at the MO62X/6-311++G(d,p) level, and we have actually found that the strenght of the $\mathrm{CH}_{3} / \pi$ interaction follows the same order found for $\pi-\pi$ stacking. The superior performance of indole over benzofuran and thiophene is thus probably due to this effect, which is likely very general when comparing the interactions of such compounds with biomolecules. Moreover, the indole system is also capable to act as an acceptor in $\pi$-acceptor hydrogen bonding, and we find a couple of interactions, where the donors are the backbone NH of Asp29 and Asp30. These interactions replace the hydrogen bonding ones given by the backbone $\mathrm{NH}$ of Ala28 and Asp29 towards the oxygens in Darunavir (Fig 3a and 3b).

A further point in favor of $\mathbf{9 b}$ in comparison with Darunavir may be given by the benzyl side chain that replaces the alkyl chain of the drug at P1'. The aromatic side chain seems actually able to establish more favorable hydrophobic interactions (see the supplementary figures S2 and S3 with the maps of the recognized interactions). This may also explain the better performance of the set of inhibitors reported in the present paper in comparison with other set previously described by us (namely the difference between $9 \mathbf{c}$ and 1c). 
As to the minor effect given by the substituents at P2', a very simple explanation is found in the relatively small size of the S2' subsite, which can fit well the aromatic ring with one methoxy group, but is unable to host both the 3,4-dimethoxyphenly or the 4-nitrophenyl groups without suffering from conformational distortions of the ligands (Supplementary figure S1).

\section{Conclusion}

In conclusion, all the newly synthesized molecules with pseudo-symmetric hydroxyethylamine core proved to be active, with excellent $\mathrm{IC}_{50}$ values and with several interaction with the enzime site. Thus, we can highlight that the presence of a bis benzyl in the core can give rigidity to the molecules and maximize the interaction. Furthermore the indole ring is apparently the heterocycle which confers greater metabolic stability, this makes the inhibitor 9a, 9b and $9 \mathbf{c}$ very promising molecules, regardless of the nature of the substituent present on the sulfonamide.

\section{Acknowledgments}

Financial support has been provided by MIUR (Italian Ministry of University) PON Ricerca e Innovazione 2014-2020-Area SALUTE -ARS01 01081,"Prodotti INnovativi ad alto contenuto biotecnologico per il settore BIOMEDicale” (INBIOMED) and University of Basilicata.

Supporting information: Copy of ${ }^{1} \mathrm{H}$ and ${ }^{13} \mathrm{C}$ NMR spectra of compounds $5 \mathbf{c}, \mathbf{6 c}, 7 \mathbf{a}-\mathbf{c}$, 8a-c and 9a-c, inhibition assays and model structures of complex of compound $\mathbf{9 b}$ and Darunavir with the protease are available.

Conflicts of Interest: The author declares no conflict of interest.

\section{References}

1. Global Report: UNAIDS report on the global AIDS epidemic 2021. https://www.unaids.org/sites/default/files/media_asset/2021-global-aids-update_en.pdf.

2. Wensing, A. M. J.; Van Maarseveen, N. M.; Nijhuis, M. Fifteen years of HIV Protease Inhibitors: raising the barrier to resistance, Antiviral Res. 2010, 85, 59-74.

3. https://www.who.int/hiv/pub/arv/chapter4.pdf.

4. Ghosh, A.K.; Chapsal BD. Aspartic acid proteases as therapeutic targets. In: Ghosh AK, editor. Methods and Principles in Medicinal Chemistry, Weinheim: Wiley-VCH; 2010, Volume 45, 169-204.

5. Tomasselli, A.G.; Heinrikson, R. L. Targeting the HIV-protease in AIDS therapy: a current clinical perspective. Biochim. et Biophys. Acta 2000, 1477, 189-214. 
6. Brik, A.; Wong, C. H. HIV-1 protease: mechanism and drug discovery. Org. Biomol. Chem. 2003, $1,5-14$.

7. Ghosh, A. K.; Rao, K. V.; Nyalapatla, P.R.; Kovela, S.; Brindisi, M.; Osswald, H. L.; Reddy, B. S.; Agniswamy, J.; Wang, Y. -F.; Aoki, M.; Hattori, S.-I.; Weber, I. T.; Mitsuya, H. Design of Highly Potent, Dual-Acting and Central-Nervous-System-Penetrating HIV-1 Protease Inhibitors with Excellent Potency against Multidrug-Resistant HIV-1 Variants ChemMedChem 2018, 13, 803-815.

8. Ghosh, A. K.; Williams, J. N.; Ho, R. Y.; Simpson H. M.; Hatton, S.-I.; Hayashi, H.; Agniswamy, J.; Wang, Y.-F.; Weber, I. T. Design and Synthesis of Potent HIV-1 Protease Inhibitors Containing Bicyclic Oxazolidinone Scaffold as the P2 Ligands: StructureActivity Studies and Biological and X-ray Structural Studies. J. Med. Chem. 2018, 61, 9722-9737.

9. Sun, S.; Jia, Q.; Zhang, Z. Applications of amide isosteres in medicinal chemistry. Bioorg. Med. Chem. Lett. 2019, 29, 2535-2550.

10. Bonini, C.; Chiummiento, L.; De Bonis, M.; Funicello, M.; Lupattelli, P.; Pandolfo,R. Application of Sharpless asymmetric dihydroxylation to thienyl- and benzothienyl acrylates and crotonates. Tetrahedron: Asymmetry 2006, 17, 2919-2924.

11. Bochicchio, A.; Cefola, R.; Choppin, S.; Colobert, F.; Di Noia, M. A.; Funicello, M.; Hanquet, G.; Pisano, I.; Todisco, S. Chiummiento, L. Selective Claisen rearrangement and iodination for the synthesis of polyoxygenated allyl phenol derivatives. Tetr. Lett. 2016, 57, 4053-4055.

12. Cerminara, I.; Chiummiento, L.; Funicello, M.; Guarnaccio, A.; Lupattelli, P. Heterocycles in Peptidomimetics and Pseudopeptides: Designand Synthesis. Pharmaceuticals 2012, 5, 297-316.

13. Bonini, C.; Chiummiento, L.; De Bonis, M.; Di Blasio, N.; Funicello, M.; Lupattelli, P.; Pandolfo, R.; Tramutola, F.; Berti, F. Synthesis of New Thienyl Ring Containing HIV-1 Protease Inhibitors: Promising Preliminary Pharmacological Evaluation against Recombinant HIV-1 Proteases. J Med Chem. 2010, 53, 1451-1457.

14. Bonini, C.; Chiummiento, L.; De Bonis, M.; Funicello, M.; Lupattelli, P.; Suanno, G.; Berti, F.; Campaner, P. Synthesis, biological activity and modelling studies of two novel anti HIV PR inhibitors with a thiophene containing hydroxyethylamino core. Tetrahedron 2005, 61, 6580-6589. 
15. Bonini, C.; Chiummiento, L.; De Bonis, M.; Funicello, M.; Lupattelli, P. Synthesis of a First Thiophene Containing Analog of the HIV Protease Inhibitor Nelfinavir. Tetrahedron Lett. 2004, 45, 2797-2799.

16. Chiummiento, L.; Funicello, M.; Lupattelli, P.; Tramutola, F.; Campaner, P. New indolic non-peptidic HIV protease inhibitors from $(S)$-glycidol: synthesis and preliminary biological activity Tetrahedron 2009, 65, 5984-5989.

17. Chiummiento, L.; Funicello, M.; Lupattelli, P.; Tramutola, F.; Berti, F.; Marino-Merlo, F. Synthesis and biological evaluation of novel small non-peptidic HIV-1 PIs: the benzothiophene ring as an effective moiety. Bioorg Med Chem Lett. 2012, 22, 2948-2950.

18. Bonini C, Chiummiento L, Di Blasio N, Funicello M., Lupattelli P., Tramutola F., Berti F., Ostric A., Miertus S., Frecer V., Kong D.-X. Synthesis and biological evaluation of new simple indolic non peptidic HIV Protease inhibitors: the effect of different substitution patterns. Bioorg. Med. Chem. 2014, 22, 4792-4802.

19. Ghosh, A.K.; Xu, C.X.; Rao, K.V.; Baldridge, A.; Agniswamy, J.; Wang, Y.F.; Weber, I.T.; Aoki, M.; Miguel, S.G.P.; Amano, M.; Mitsuya, H. Probing multidrug-resistance/proteinligand interaction with oxatricyclic designed ligands in HIV-1 Protease inhibitors. ChemMedChem 2010, 5, 1850-1854.

20. Ghosh, A.K.; Anderson, D.D.; Weber, I.T.; Mitsuya, H. Enhancing Protein Backbone Binding-A Fruitful Concept for Combating Drug-Resistant HIV. Angew. Chem. Int. Ed. Engl. 2012, 51, 1778-1802.

21. Zhang, H.; Wang, Y.F.; Shen, C.H.; Agniswamy, J.; Rao, K.V.; Xu, X.; Ghosh, A.K.; Harrison, R.W.; Weber, I.T. Novel P2 Tris-tetrahydrofuran Group in Antiviral Compound 1 (GRL-0519) Fills the S2 Binding Pocket of Selected Mutants of HIV-1 Protease. J. Med. Chem. 2013, 56, 1074-1083.

22. Funicello, M.; Chiummiento, L.; Tramutola, F.; Armentano, M. F.; Bisaccia, F.; Miglionico, R.; Milella, L.; Benedetti, F.; Berti, F.; Lupattelli, P. Synthesis and biological evaluation in vitro and in mammalian cells of new heteroaryl carboxyamides as HIVprotease inhibitors. Bioorg.Med. Chem. 2017, 25, 4715-4722.

23. Tramutola, F.; Armentano, M. F.; Berti, F.; Chiummiento, L.; Lupattelli, P.; D'Orsi, R.; Miglionico, R.; Milella, L.; Bisaccia, F.; Funicello, M. New heteroaryl carbamates: synthesis and biological screening in vitro and in mammalian cells of wild-type and mutant HIV-protease inhibitors. Bioorg. Med. Chem. 2019, 27, 1863-1870.

24. Hidaka, K.; Kimura,T.; Hayashi, Y.; McDaniel, K. F.;Dekhtyar, T.; Colletti, L.;Kiso, Y. Design and Synthesis of Pseudo-Symmetric HIV Protease Inhibitors Containing a Novel 
Hydroxymethylcarbonyl (HMC)-Hydrazide Isostere. Bioorg. Med. Chem. Lett. 2003, 13, 93-96.

25. Facchinetti, V.; Moreth, M.; Gomes, C. R. B.; do O'Pessoa, C.; Rodrigues, F. A. R.; Cavalcanti, B. C.; Oliveira, A. C. A.; Carneiro, T. R.; Gama, I. L.; de Souza, M. V. N. Evaluation of (2S,3R)-2-(amino)-[4-(N-benzylarenesulfonamido)-3-hydroxy-1phenylbutane derivatives: a promising class of anticancer agents Med. Chem. Res. 2015, 24, 533-542.

26. Schrödinger Release 2021-3: MacroModel, Schrödinger, LLC, New York, NY, 2021] The inhibitors were then docked inside the catalytic site of the enzyme with the Schrödinger Glide protocol.

27. Friesner, R. A.; Murphy, R. B.; Repasky, M. P.; Frye, L. L.; Greenwood, J. R.; Halgren,T. A.; Sanschagrin, P. C.; Mainz, D. T. Extra Precision Glide: Docking and Scoring Incorporating a Model of Hydrophobic Enclosure for Protein-Ligand Complexes. J. Med. Chem. 2006, 49, 6177-6196.

28. Trucks, G.W.; Frisch, M.J.; Schlegel, H.B.; Scuseria, G.E.; Robb, M.A.; Cheeseman, J.R.; Scalmani, G.; Barone, V.; Petersson, G.A.; Nakatsuji, H.; Li, X.; Caricato, M.; Marenich, A.; Bloino, J.; Janesko, B.G.; Gomperts, R.; Mennucci, B.; Hratchian, H.P.; Ortiz, J.V.; Izmaylov, A.F.; Sonnenberg, J.L.; Williams-Young, D.; Ding, F.; Lipparini, F.; Egidi, F.; Goings, J.; Peng, B.; Petrone, A.; Henderson, T.; Ranasinghe, D.; Zakrzewski, V.G.; Gao, J.; Rega, N.; Zheng, G.; Liang, W.; Hada, M.; Ehara, M.; Toyota, K.; Fukuda, R.; Hasegawa, J.; Ishida, M.; Nakajima, T.; Honda, Y.; Kitao, O.; Nakai, H.; Vreven, T.; Throssell, K.; Montgomery, J.A. Jr.; Peralta, J.E.; Ogliaro, F.; Bearpark, M.; Heyd, J.J.; Brothers, E.; Kudin, K.N.; Staroverov, V.N.; Keith, T.; Kobayashi, R.; Normand, J.; Raghavachari, K.; Rendell, A.; Burant, J.C.; Iyengar, S.S.; Tomasi, J.; Cossi, M.; Millam, J.M.; Klene, M.; Adamo, C.; Cammi, R.; Ochterski, J.W.; Martin, R.L.; Morokuma, K.; Farkas, O.; Foresman, J.B.; Fox, D.J, (2009) Gaussian 09, Gaussian, Inc., Wallingford CT, 2016.

29. Bai X., Yang Z., Zhu M., Dong B., Zhu L., Zhang G., Wang J., Wang Y. Design and synthesis of potent HIV-1 protease inhibitors with $(S)$-tetrahydrofuran-tertiary amineacetamide as P2ligand: Structure activity studies and biological evaluation. Eur. J. Med. Chem. 2017, 137, 30-44.

30. Yang Z.-H., Bai X.-G., Zhou, L., Wang J.-X., Liu H.-T., Wang Y.-C. Synthesis and biological evaluation of novel HIV-1 protease inhibitors using tertiary amine as P2-ligands. Bioorg. Med. Chem. Lett. 2015, 25, 1880-1883. 
31. Chow, W.A.; Jiang, C.; Guan, M. Anti-HIV drugs for cancer therapeutics: back to the future? The Lancet 2009, 10, 61-71.

32. Toupkanloo, H.A., Rahmani, Z. An in-depth study on non-covalent stacking interactions between DNA bases and aromatic drug fragments using DFT method and AIM analysis: conformers, binding energies, and charge transfer. App. Biol. Chem. 2018, 61, 209-226. 